\title{
NEW WEIGHTED ČEBYŠEV-OSTROWSKI TYPE INTEGRAL INEQUALITIES ON TIME SCALES
}

\author{
ADNAN TUNA AND WENJUn LiU
}

Abstract. In this paper we obtain some weighted Čebyšev-Ostrowski type integral inequalities on time scales involving functions whose first derivatives belong to $L_{p}(a, b)(1 \leqslant p \leqslant \infty)$. We also give some other interesting inequalities as special cases.

Mathematics subject classification (2010): 26D15, 26E70.

Keywords and phrases: Weighted Čebyšev-Ostrowski type inequality, weighted Čebyšev type inequality, time scales.

\section{REFERENCES}

[1] R. Agarwal, M. Bohner, A. Peterson, Inequalities on time scales: a survey, Math. Inequal. Appl., 4 (2001), 535-557.

[2] F. Ahmad, N. M. Mir, A. RAFIQ, Weighted Čebyšev Type inequalities involving functions whose first derivatives belong to $L_{p}(a, b), \forall(1 \leqslant p<\infty)$, Kragujevac J. Math. 32 (2009), 13-26.

[3] G. Anastassiou, Duality principle of time scales and inequalities, Appl. Anal., 89 (2010), 1837 1854.

[4] M. Bohner, A. Peterson, Dynamic Equations on Time Scales, An Introduction with Applications, Birkhäuser Boston, Inc., Boston, MA, 2001.

[5] M. Bohner, A. Peterson, Advances in Dynamic Equations on Time Scales, Birkhäuser Boston, Boston, MA, 2003.

[6] M. Bohner, T. Matthews, The Grüss inequality on time scales, Commun. Math. Anal., 3 (2007), $1-8$.

[7] M. Bohner, T. Matthews, Ostrowski inequalities on time scales, JIPAM. J. Inequal. Pure Appl. Math., 9 (2008), Article 6, 8 pp.

[8] M. Bohner, T. Matthews, A. Tuna, Diamond-alpha Grüss type inequalities on time scales, Int. J. Dyn. Syst. Differ. Equ., 3 (2011), 234-247.

[9] M. Bohner, T. Matthews, A. TunA, Weighted Ostrowski-Grüss inequalities on time scales, African Diaspora Journal of Mathematics, Volume 12, Number 1, 2011, 89-99.

[10] C. Dinu, Ostrowski type inequalities on time scales, An. Univ. Craiova Ser. Mat. Inform., 34 (2007), 43-58.

[11] S. HILGER, Ein Maßkettenkalkül mit Anwendung auf Zentrumsmannigfaltigkeiten, Ph. D. thesis, Universität Würzburg, Würzburg, Germany, 1988.

[12] B. KARPUZ, U. M. OZKAN, Generalized Ostrowski's inequality on time scales, JIPAM. J. Inequal. Pure Appl. Math., 9 (2008), Article 112, 7 pp.

[13] V. Lakshmikantham, S. Sivasundaram, B. Kaymakcalan, Dynamic Systems on Measure Chains, Mathematics and its Applications, 370, Kluwer Academic Publishers Group, Dordrecht, 1996.

[14] W. J. LiU, Q.-A. NGô, An Ostrowski-Grüss type inequality on time scales, Comput. Math. Appl., 58 (2009), 1207-1210.

[15] W. J. LiU, Q.-A. NGÔ, A generalization of Ostrowski inequality on time scales for $k$ points, Appl. Math. Comput., 203 (2008), 754-760.

[16] W. J. LiU, Q.-A. NGô, W. B. CHEN, A perturbed Ostrowski-type inequality on time scales for $k$ points for functions whose second derivatives are bounded, J. Inequal. Appl., 2008, Art. ID 597241, $12 \mathrm{pp}$. 
[17] W. J. LiU, Q.-A. NGô, W. B. Chen, A new generalization of Ostrowski type inequality on time scales, An. Ştiinț. Univ. "Ovidius" Constanţa, 17 (2009), 101-114.

[18] W. J. Liu, A. TunA, Weighted Ostrowski, trapezoid and Grüss type inequalities on time scales, J. Math. Inequal., 6 (2012), 381-399.

[19] Q.-A. NGô, W. J. LIU, A sharp Grüss type inequality on time scales and application to the sharp Ostrowski-Grüss inequality, Commun. Math. Anal., 6 (2009), 33-41.

[20] A. Ostrowski, Uber die Absolutabweichung einer differentiierbaren Funktion von ihrem Integralmittelwert, Comment. Math. Helv., 10 (1937), 226-227.

[21] A. RAFIQ, A. M. Mir, F. Ahmad, Weighted Čebyšev-Ostrowski type inequalities, Applied mathematics and Mechanics (English Edition), 28 (7) (2007), 901-906.

[22] M.Z. S ARIKAYA, A note on Grüss type inequalities on time scales, Dynam. Systems Appl., 17 (2008), 663-666.

[23] M. Z. SARIKAYA, New weighted Ostrowski and Čebyšev type inequalities on time scales, Computers \& Mathematics with Applications, Volume 60 Issue 5, September, 2010, 1510-1514.

[24] M. Z. Sarikaya, N. AKtan, H. Yildirim, On weighted Čebyšev-Grüss type inequalities on time scales, J. Math. Inequal., 2 (2008), 185-195.

[25] M. R. Sidi Ammi, D. F. M. Torres, Combined dynamic Grüss inequalities on time scales, J. Math. Sci. (N. Y.), 161 (2009), 792-802.

[26] A. Tuna, D. Daghan, Generalization of Ostrowski and Ostrowski-Grüss type inequalities on time scales, Comput. Math. Appl., 60 (2010), 803-811.

[27] A. Tuna, Y. JiAng, W. J. Liu, Weighted Ostrowski, Ostrowski-Grüss and Ostrowski-Čebyšev Type Inequalities on Time Scales, Publ. Math. Debrecen, 81 (2012), 81-102.

[28] C. C. Yen, F. H. Wong, H. J. LI, Čebyšev's inequality on time scales, Journal of Inequalities in Pure and Applied Mathematics, Volume 6, Issue 1, Article 7, 2005. 